\title{
Lipschitz Continuous Ordinary Differential Equations are Polynomial-Space Complete
}

\author{
Akitoshi Kawamura
}

April 2010

\begin{abstract}
In answer to Ko's question raised in 1983, we show that an initial value problem given by a polynomial-time computable, Lipschitz continuous function can have a polynomial-space complete solution. The key insight is simple: the Lipschitz condition means that the feedback in the differential equation is weak. We define a class of polynomial-space computation tableaux with equally weak feedback, and show that they are still polynomial-space complete. The same technique also settles Ko's two later questions on Volterra integral equations.
\end{abstract}

Keywords: computable analysis; computational complexity; initial value problem; Lipschitz condition; ordinary differential equations; Picard-Lindelöf Theorem; polynomial space.

\section{Introduction}

Let $g:[0,1] \times \mathbf{R} \rightarrow \mathbf{R}$ be a continuous function and consider the initial value problem

$$
h(0)=0, \quad h^{\prime}(t)=g(t, h(t)), \quad t \in[0,1] .
$$

A well-known sufficient condition (see the beginning of Section 3 for a proof sketch) for this equation to have a unique solution $h:[0,1] \rightarrow \mathbf{R}$ is that $g$ be Lipschitz continuous (in its second argument), i.e.,

$$
\left|g\left(t, y_{0}\right)-g\left(t, y_{1}\right)\right| \leq Z \cdot\left|y_{0}-y_{1}\right|, \quad t \in[0,1], y_{0}, y_{1} \in \mathbf{R}
$$

for some constant $Z$ independent of $y_{0}, y_{1}$ and $t$. We are interested in the computational complexity of the solution $h$ under this condition.

Our model of computation of real functions, which will be reviewed in Section 2, is adopted from computable analysis and is thus consistent with the conventional notion of computability. We formulate our main result in Section 3: the solution $h$ of the above equation can be polynomial-space complete, even if $g$ is polynomial-time computable. This was open since Ko (1983). The essential part of the proof is given in Section 4, where we construct a certain family of real functions that can be used as building blocks for the desired 
$g$ and $h$. The main idea is to regard the differential equation with the Lipschitz condition as a polynomial-space computation tableau with some restrictions. In Section 5, we state a few variants of the main theorem, two of which solve the problems about Volterra integral equations posed by Ko (1992). These variants are also proved using the same building blocks, as shown in Section 6. Section 7 discusses related results and open problems.

\section{Computational complexity of real functions}

The study of mathematical analysis from the viewpoint of computability is called computable analysis; Brattka et al. (2008) and Weihrauch (2000) are good introductions to the field. We review the basic definitions briefly here, refining them for our complexity consideration where necessary.

The computability notion for real functions equivalent to ours dates back at least to Grzegorczyk (1955). Polynomial-time computability of real functions was introduced by Ko \& Friedman (1982) using oracle machines, and is equivalent, at least in our context, to the one defined by the type-two machine and the signed digit representation (Chapter 7 of Weihrauch (2000)).

\subsection{Computing real functions}

Since real numbers cannot be encoded into strings, we encode them into functions from strings to strings. We say that a real number $t$ is represented by a string function $A$ if for any $m \in \mathbf{N}$, the string $A\left(0^{m}\right)$ is the binary notation (with a sign bit at the beginning) of either $\left\lfloor 2^{m} t\right\rfloor$ or $\left\lceil 2^{m} t\right\rceil$, where $\lfloor\cdot\rfloor$ and $\lceil\cdot\rceil$ mean rounding down and up to the nearest integer, respectively. In effect, $A\left(0^{m}\right)$ gives an approximation of $t$ with precision $2^{-m}$ by a multiple of $2^{-m}$. We also say that $A$ is a name of $t$.

Computation of real functions is realized by oracle Turing machines (henceforth just machines) working on such names $A$. In addition to the input, output and work tapes, the machine has a query tape and can consult an external oracle $A$ by entering a distinguished state; the string $v$ which is on the query tape at this moment is then replaced by $A(v)$ in one step. We write $M^{A}$ for the string-to-string function computed by machine $M$ with oracle $A$.

Definition 2.1. A machine $M$ computes a function $f:[0,1] \rightarrow \mathbf{R}$ if for any $t \in[0,1]$ and any name $A$ of it, $M^{A}$ is a name of $f(t)$.

Thus, computation of a real function $f$ is a Turing reduction of (a name of) $f(t)$ to $t$ (Figure 2.1, left). A little thought shows that it can equivalently be visualized as a Turing machine that, given on the input tape an infinite sequence of approximations of $t$, writes approximations of $f(t)$ endlessly on the one-way output tape (Figure 2.1, right).

A machine runs in polynomial time if there is a polynomial $p: \mathbf{N} \rightarrow \mathbf{N}$ such that, for any input string $u$, it halts within $p(|u|)$ steps regardless of the oracle. A real function is (polynomial-time) computable if some machine (that runs in polynomial time) computes it.

When writing an approximation of $f(t)$ with precision $2^{-m}$, the machine knows $t$ only to some finite precision $2^{-n}$. Hence, all computable functions are continuous. If the machine 

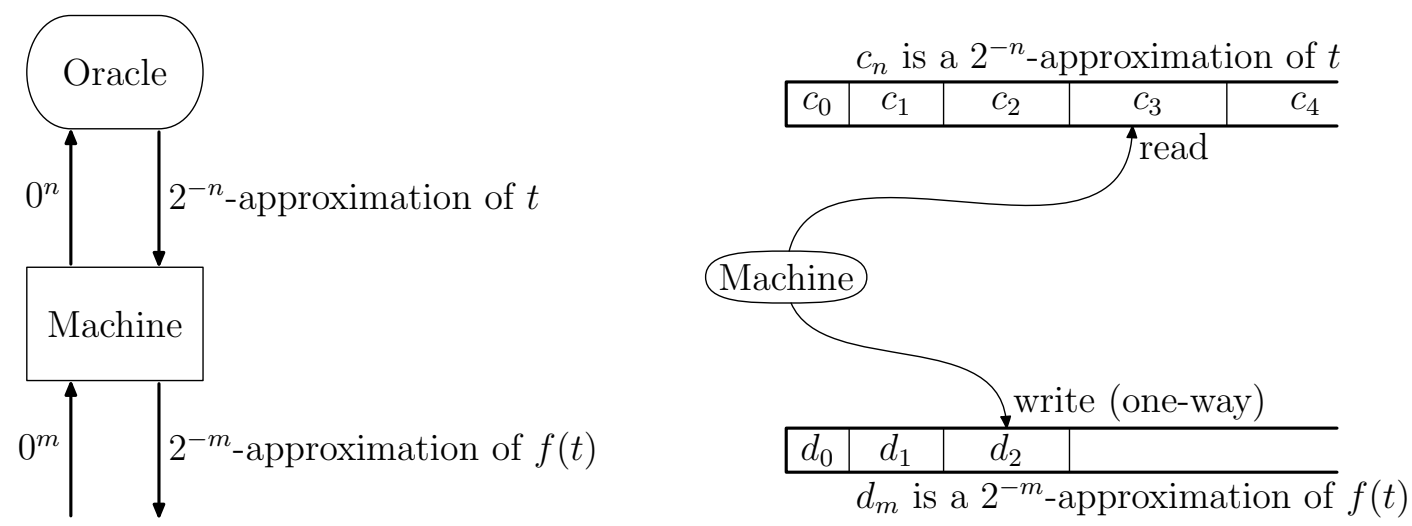

Figure 2.1: To compute a real function $f$, the machine should output an approximation of $f(t)$ with given precision $2^{-m}$ by consulting the oracle for approximations of $t$ with any precision $2^{-n}$ it desires (left). An alternative picture (right) is that the machine converts any stream of improving approximations of $t$ to a stream of improving approximations of $f(t)$.

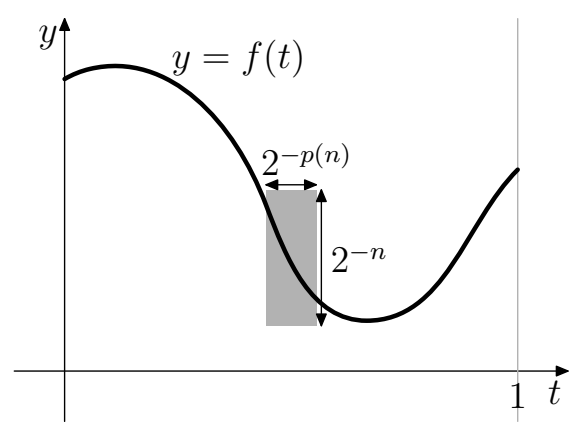

Figure 2.2: Modulus of continuity $p$.

runs in polynomial time, then this $n$ is bounded polynomially in $m$. Hence, all polynomialtime computable functions $f$ have a polynomial modulus of continuity (Figure 2.2): there is a polynomial $p$ such that $\left|f\left(t_{0}\right)-f\left(t_{1}\right)\right|<2^{-m}$ for all $t_{0}, t_{1} \in[0,1]$ and $m \in \mathbf{N}$ with $\left|t_{0}-t_{1}\right|<2^{-p(m)}$ (note that in our setting it makes sense to put $p$ in the exponent, deviating from some authors' terminology where a modulus of continuity means a function that takes an upper bound on $\left|t_{0}-t_{1}\right|$ to that on $\left.\left|f\left(t_{0}\right)-f\left(t_{1}\right)\right|\right)$. In fact, it is not hard to see that polynomial-time computability can be characterized by this plus the assertion that $f$ can be approximated at rationals:

Lemma 2.2. A function $f:[0,1] \rightarrow \mathbf{R}$ is polynomial-time computable if and only if it has a polynomial modulus of continuity and there is a polynomial-time computable function $g:([0,1] \cap \mathbf{Q}) \times\{0\}^{*} \rightarrow \mathbf{Q}$ such that

$$
\left|g\left(d, 0^{n}\right)-f(d)\right|<2^{-n}, \quad d \in[0,1] \cap \mathbf{Q}, n \in \mathbf{N}
$$

where rational numbers are encoded in a reasonable way (e.g., using fractions whose numerator and denominator are integers written in binary). 
Many familiar continuous functions are computable. For example, it is easy to see that the sine function restricted to $[0,1]$ is polynomial-time computable, because an approximation of

$$
\sin t=t-\frac{t^{3}}{3 !}+\frac{t^{5}}{5 !}-\frac{t^{7}}{7 !}+\cdots
$$

to precision $2^{-m}$ can be found by approximating the sum of polynomially many (in $m$ ) initial terms, since this series converges fast enough on $[0,1]$.

The above definition can be straightforwardly extended to functions on compact intervals other than $[0,1]$ and on $d$-dimensional rectangles (by considering machines taking $d$ oracles). Also, polynomial-space, exponential-time and exponential-space computability is defined analogously to polynomial-time computability, where by "exponential" we mean $2^{n^{\mathrm{O}(1)}}$ (and not $2^{\mathrm{O}(n)}$ ). Here, we count the query tape in when defining space complexity. The definition in Section 7.2.1 of Ko (1991) states to the contrary, but his subsequent theorems build on the definition that does charge the query tape (on the other hand, his argument in Chapter 4 that the query tape should not be counted in discussing logarithmic space is correct).

\subsection{Completeness}

We now introduce terminology to state our main results which say that certain real functions are "hard" to compute. We regard a language $L$ as a set of strings or as a $\{0,1\}$-valued function interchangeably, so that $L(u)=1$ means $u \in L$. We write $\mathbf{P}, \mathbf{N P}$, PSPACE, EXPTIME, EXPSPACE for the standard classes of languages and \# $\mathbf{P}$ for the function class; see Papadimitriou (1994).

Definition 2.5. A function $L$ (over strings) is said to reduce to a real function $h:[0,1] \rightarrow$ $\mathbf{R}$ if the following holds for some polynomial-time computable functions $R, S, T$ : Let $u$ be a string, and suppose that the function taking string $v$ to $S(u, v)$ is a name of a real number $t \in[0,1]$. Then $L(u)=R(u, \psi(T(u)))$ for any name $\psi$ of $f(t)$ (Figure 2.3).

For a complexity class $\mathcal{C}$, we say that a real function is $\mathcal{C}$-hard if all problems in $\mathcal{C}$ reduce to it. A real function is polynomial-space (resp. exponential-space) complete if it is polynomial-space (resp. exponential-space) computable and PSPACE-hard (resp. EXPSPACE-hard).

The above definitions of reduction and completeness can be viewed as a special case of those by Beame et al. (1998), and are also consistent with Definition 2.2 of Ko (1992).

\section{Ko's question and our main result}

Now we return to the differential equation (1.1). The fact that (1.2) guarantees a unique solution is known as (a variant of) the Picard-Lindelöf (or Cauchy-Lipschitz) Theorem, and can be proved as follows. Let $C$ be the set of all continuous real functions on $[0,1]$. A 


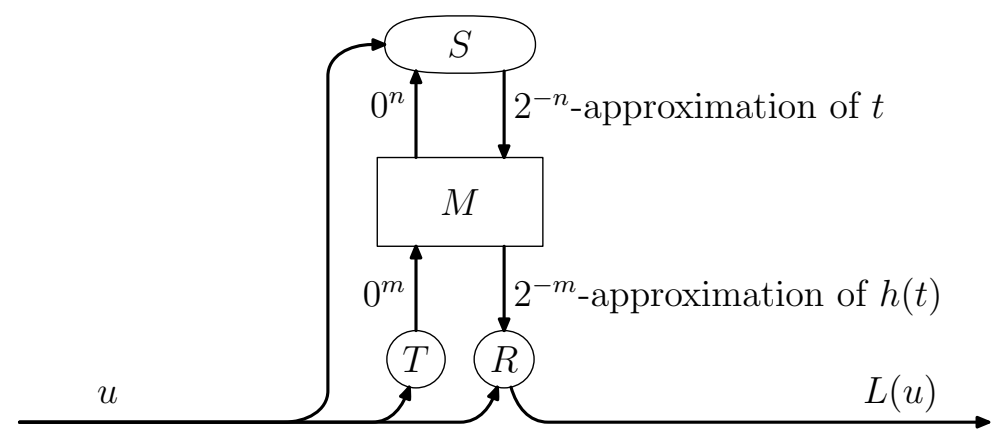

Figure 2.3: $L$ reduces to $h$ via $R, S$ and $T$. This means that, using a hypothetical machine $M$ computing $h$ (in the sense of Definition 2.1) as a black box, we can compute $L$ in polynomial time in the way depicted above.

solution of (1.1) is a fixed point of the operator $T: C \rightarrow C$ defined by

$$
T(h)(t)=\int_{0}^{t} g(\tau, h(\tau)) \mathrm{d} \tau, \quad t \in[0,1] .
$$

The existence and uniqueness of this fixed point follow from the contraction principle (Banach fixed point theorem), because a simple calculation shows that, for the metric $d$ on $C$ given by $d\left(h_{0}, h_{1}\right)=\max _{t \in[0,1]} \exp (-2 Z t)\left|h_{0}(t)-h_{1}(t)\right|$, we have $d\left(T\left(h_{0}\right), T\left(h_{1}\right)\right) \leq$ $d\left(h_{0}, h_{1}\right) / 2$.

We assume the following, and ask how complex $h$ can be:

(*) $g:[0,1] \times \mathbf{R} \rightarrow \mathbf{R}$ and $h:[0,1] \rightarrow \mathbf{R}$ satisfy (1.1), $g$ satisfies (1.2), and $g$ is polynomialtime computable.

Strictly speaking, we have defined polynomial-time computability only for functions on a compact rectangle. What we mean here is that the restriction of $g$ to $[0,1] \times[\min h, \max h]$, say, is polynomial-time computable. Equivalently, we could write $g:[0,1] \times[-1,1] \rightarrow \mathbf{R}$ and add the clause "the values of $h$ stays within $[-1,1]$ " to $(*)$; this does not essentially change our result, because we can always make $h$ stay within $[-1,1]$ by scaling $g$ and $h$ down by a constant factor, which does not affect polynomial-time computability. There is a way to extend Definition 2.1 to functions with unbounded domain, as in Hoover (1990) or pp. 57-58 of Ko (1991), but we choose our simpler definition.

As Ko (1983) points out by analyzing the Euler method, (*) implies that $h$ is polynomialspace computable. From this it follows (Lemma 2.2 of Ko (1983)) that if $\mathbf{P}=$ PSPACE, then $(*)$ implies that $h$ is polynomial-time computable. We will prove a lower bound that matches this upper bound:

Theorem 3.2. There are functions $g$ and $h$ satisfying $(*)$ such that $h$ is PSPACE-hard (and thus polynomial-space complete). 
Corollary 3.3. $\mathbf{P}=$ PSPACE if and only if $(*)$ always implies that $h$ is polynomialtime computable.

This solves the main problem left open in Ko (1983). He had proved there a partial result essentially stating that Theorem 3.2 holds true if the Lipschitz condition (1.2) in the assumption $(*)$ is replaced by a weaker condition.

We remark that the special case of the equation (1.1) where $g$ ignores its second argument reduces to integration, whose complexity is summarized as follows in the style similar to Theorem 3.2 and Corollary 3.3.

THEOREM 3.4 (essentially by Friedman 1984). There are a polynomial-time computable function $g:[0,1] \rightarrow \mathbf{R}$ and a \#P-hard function $h:[0,1] \rightarrow \mathbf{R}$ such that

$$
h(t)=\int_{0}^{t} g(\tau) \mathrm{d} \tau, \quad t \in[0,1] .
$$

Corollary 3.6 (Friedman 1984). $\mathbf{P}=\mathbf{P}^{\# \mathbf{P}}$ if and only if for all polynomial-time computable $g:[0,1] \rightarrow \mathbf{R}$, the function $h$ defined by (3.5) is polynomial-time computable.

The relation to the counting class is not surprising: as is apparent from Friedman's proof, the intuition behind this result is that approximating the integral is to count the number of grid points below the graph of $g$.

This bound of $\mathbf{P}^{\# \mathbf{P}}$ has been the best known lower bound also for our differential equation. Theorem 3.2 improves this to PSPACE.

\section{Proof of the theorem}

We present the proof backwards, reducing Theorem 3.2 to Lemma 4.1 and then reducing Lemma 4.1 to Lemma 4.7. In Section 4.1, we state Lemma 4.1 asserting the existence of a certain family of pairs of functions $\left(g_{u}\right)_{u}$ and $\left(h_{u}\right)_{u}$, from which the functions $g$ and $h$ in Theorem 3.2 can be constructed. Section 4.2 shows that Lemma 4.1 follows from Lemma 4.7, which asserts the PSPACE-completeness of a discrete version of the initial value problem. This discrete problem is like a PSPACE computation tableau, but with a certain restriction similar to the Lipschitz condition. Section 4.3 then completes the proof by showing Lemma 4.7 .

\subsection{Building blocks}

To state Lemma 4.1, we need to extend the definition of computation in Section 2.1 to families of real functions indexed by strings $u$. This is done in the natural way, by giving $u$ as another string input to the machine. For example, a family $\left(g_{u}\right)_{u}$ of functions $g_{u}:[0,1] \times$ $[-1,1] \rightarrow \mathbf{R}$ is computed by a machine $M$ if for any names $A$ and $B$ of $t \in[0,1]$ and $y \in[-1,1]$, the function that takes string $0^{m}$ to $M^{A, B}\left(u, 0^{m}\right)$ is a name of $g_{u}(t, y)$. Note that in this case, claiming that $M$ runs in polynomial time means that it halts in time polynomial in $|u|+m$. 


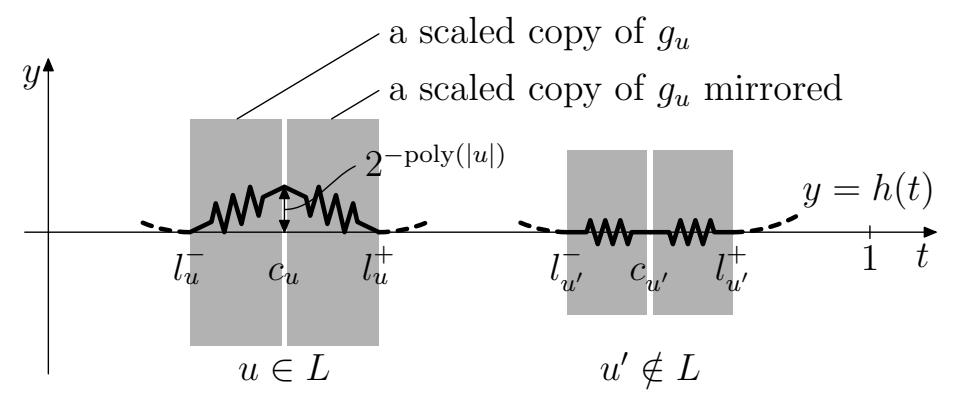

Figure 4.1: To construct $g$, we assign interval $\left[l_{u}^{-}, l_{u}^{+}\right]$to each string $u$ and put there a pair of reduced copies of $g_{u}$. The value $h\left(c_{u}\right)$ at the midpoint will be positive if and only if $u \in L$.

Lemma 4.1. Let $L \in$ PSPACE and let $\lambda: \mathbf{N} \rightarrow \mathbf{N}$ be a polynomial. Then there exist a polynomial $\rho: \mathbf{N} \rightarrow \mathbf{N}$ and families of functions $g_{u}:[0,1] \times[-1,1] \rightarrow \mathbf{R}$ and $h_{u}:[0,1] \rightarrow \mathbf{R}$ indexed by binary strings $u$ such that the family $\left(g_{u}\right)_{u}$ is polynomial-time computable and for each $u$ we have

(i) $h_{u}(t) \in[-1,1]$ for all $t \in[0,1]$;

(ii) $g_{u}(0, y)=g_{u}(1, y)=0$ for all $y \in[-1,1]$;

(iii) $h_{u}(0)=0$ and $h_{u}^{\prime}(t)=g_{u}\left(t, h_{u}(t)\right)$ for all $t \in[0,1]$;

(iv) $\left|g_{u}\left(t, y_{0}\right)-g_{u}\left(t, y_{1}\right)\right| \leq 2^{-\lambda(|u|)}\left|y_{0}-y_{1}\right|$ for any $t \in[0,1]$ and $y_{0}, y_{1} \in[-1,1]$;

(v) $h_{u}(1)=2^{-\rho(|u|)} L(u)$.

We thus have a family of functions $g_{u}$ that each give an initial value problem whose solution $h_{u}$ encodes $L(u)$ in its final value $h_{u}(1)$.

Using this family, the functions $g$ and $h$ in Theorem 3.2 will be constructed roughly as follows. Divide $[0,1)$ into infinitely many subintervals $\left[l_{u}^{-}, l_{u}^{+}\right]$, one for each $u$, with midpoints $c_{u}$. We put a pair of scaled copies of $g_{u}$ onto $\left[l_{u}^{-}, c_{u}\right]$ and $\left[c_{u}, l_{u}^{+}\right]$as shown in Figure 4.1 so that the membership of $u$ in $L$ can be determined by looking at $h\left(c_{u}\right)$. Scaling down $g_{u}$ horizontally increases its Lipschitz constant, and the resulting $g$ needs to have a Lipschitz constant independent of $u$; this is why we had to claim in (iv) that the $g_{u}$ originally have small constant $2^{-\lambda(|u|)}$. Details are routine and are relegated to Section 6 along with the proofs of two other theorems which will be stated in Section 5 and which will also follow from Lemma 4.1.

\subsection{Discrete initial value problem and the Lipschitz condition}

A first attempt to prove Lemma 4.1 would be as follows. Consider a polynomial-space Turing machine that decides whether a given string $u$ belongs to $L$. Its configuration at each time can be encoded into a nonnegative integer less than $2^{C(|u|)}$, where $C$ is a polynomial. There is a simple rule that maps $u$ (the input), $T$ (time) and $d$ (the current configuration) to a number $G_{u}(T, d)$ (the next configuration) such that the recurrence

$$
H_{u}(0)=0, \quad H_{u}(T+1)=G_{u}\left(T, H_{u}(T)\right)
$$




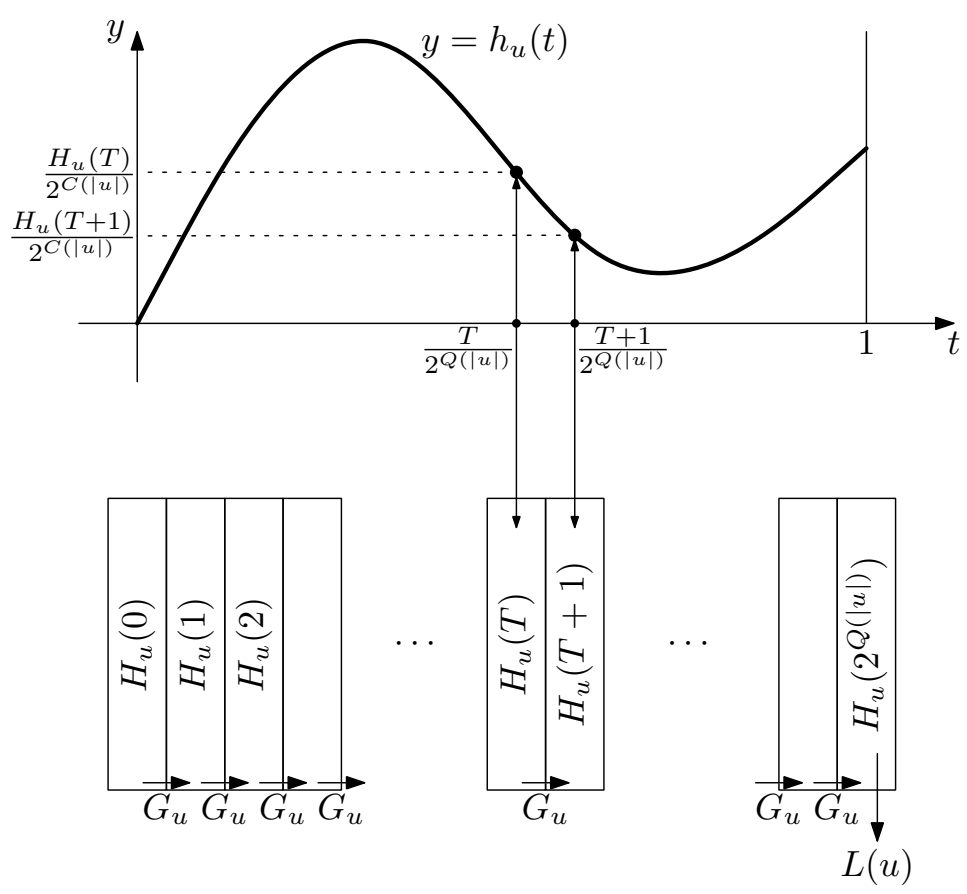

Figure 4.2: An attempt to simulate a polynomial-space Turing machine by an initial value problem is to encode the machine configuration $H_{u}(T)$ at each time $T$ into the value $h_{u}(t)=$ $H_{u}(T) / 2^{C(|u|)}$ at time $t=T / 2^{Q(|u|)}$.

leads to $H_{u}\left(2^{Q(|u|)}\right)=L(u)$ for some polynomial $Q$. Now this situation looks similar to the one in Lemma 4.1: starting at 0 , the value of $H_{u}$ (or $h_{u}$ ) changes over time according to a simpler function $G_{u}$ (or $g_{u}$ ), to reach a value eventually that indicates the answer $L(u)$. Thus we are tempted to simulate the "discrete initial value problem" (4.2) by embedding each value $H_{u}(T)$ as real number $H_{u}(T) / 2^{C(|u|)}$ (Figure 4.2).

The obstacle to this attempt is that the differential equation (iii) in Lemma 4.1 cannot express all discrete recurrences of form (4.2): continuous trajectories cannot branch or cross one another; besides, we have the Lipschitz condition (iv) that puts restriction on how strong the feedback of $h$ to itself can be. We thus need to restrict the discrete problem (4.2) so that it can be simulated by the continuous version.

To do so, let us reflect on what the Lipschitz condition (iv) means. A rough calculation shows that if two trajectories differ by $\varepsilon$ at time $t$, then they can differ at time $t+2^{-Q(|u|)}$ by at most $\varepsilon \exp \left(2^{-\lambda(|u|)} 2^{-Q(|u|)}\right) \approx \varepsilon\left(1+2^{-\lambda(|u|)-Q(|u|)}\right)$. Thus, the gap can only widen (or narrow) by a factor of $\pm 2^{-\lambda(|u|)-Q(|u|)}$ during each time interval of length $2^{-Q(|u|)}$. In other words, the feedback caused by equation (iii) is so weak that each digit of $h_{u}$ can only affect far lower digits of $h_{u}$ in the next step.

Now we define a discrete problem that reflects this restriction. Let $P$ and $Q$ be polynomials and let

$$
\begin{aligned}
& G_{u}:[P(|u|)] \times\left[2^{Q(|u|)}\right] \times[4] \rightarrow\{-1,0,1\}, \\
& H_{u}:[P(|u|)+1] \times\left[2^{Q(|u|)}+1\right] \rightarrow[4],
\end{aligned}
$$




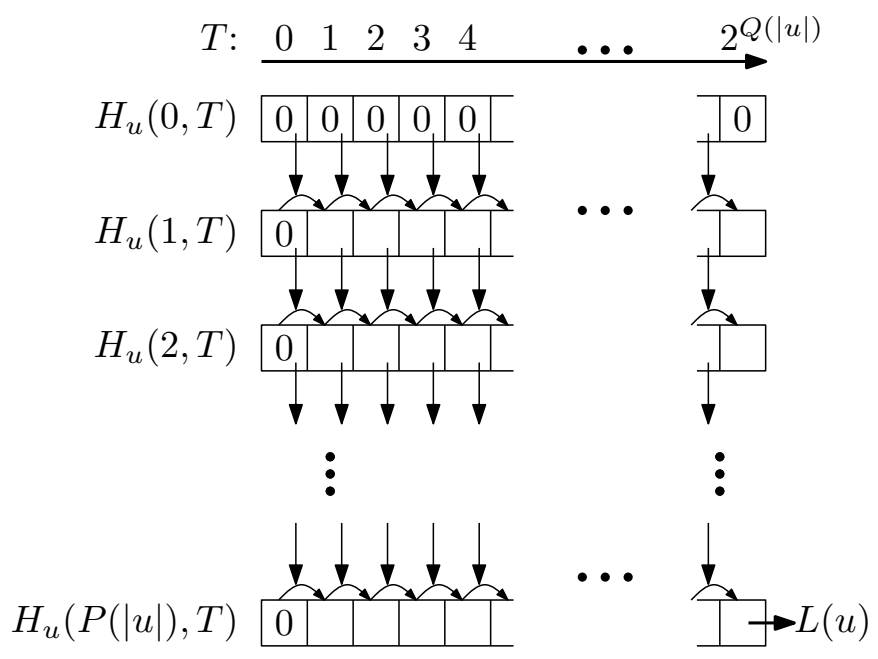

Figure 4.3: The discrete initial value problem (4.3)-(4.6). Each cell $H_{u}(T)$ in Figure 4.2 is now divided into $H_{u}(0, T), \ldots, H_{u}(P(|u|), T)$; the increment from $H_{u}(i+1, T)$ to $H_{u}(i+$ $1, T+1)$ is computed by $G_{u}$ using the upper left cell $H_{u}(i, T)$.

where we write $[N]=\{0, \ldots, N-1\}$ for $N \in \mathbf{N}$. Our restricted discrete initial value problem is as follows:

$$
\begin{aligned}
H_{u}(i, 0) & =H_{u}(0, T)=0 \\
H_{u}(i+1, T+1) & =H_{u}(i+1, T)+G_{u}\left(i, T, H_{u}(i, T)\right) .
\end{aligned}
$$

Thus, $H_{u}(T)$ of $(4.2)$ is now divided into polynomially many (in $\left.|u|\right)$ components $H_{u}(0, T)$, $\ldots, H_{u}(P(|u|), T)$; compare Figures 4.2 (bottom) and 4.3. We have added the restriction that $G_{u}$ sees only the component $H_{u}(i, T)$, which in Figure 4.3 means the upper left of the current cell. The following lemma states that, despite this restriction, we still have PSPACE-completeness. Note that making $G_{u}$ completely oblivious to its last argument would be an overkill, because then $H_{u}$ would just add up the values of $G_{u}$, resulting in the complexity merely of $\# \mathbf{P}$.

Lemma 4.7. Let $L \in$ PSPACE. Then there are polynomials $P, Q$ and families $\left(G_{u}\right)_{u}$, $\left(H_{u}\right)_{u}$ satisfying (4.3)-(4.6) such that $\left(G_{u}\right)_{u}$ is polynomial-time computable and $H_{u}(P(|u|)$, $\left.2^{Q(|u|)}\right)=L(u)$ for each string $u$.

Before proving this, we will reduce Lemma 4.1 to Lemma 4.7 by simulating the new system (4.3)-(4.6) by the differential equation. Using $G_{u}$ and $H_{u}$ of Lemma 4.7, we will construct $g_{u}$ and $h_{u}$ of Lemma 4.1 such that $h_{u}\left(T / 2^{Q(|u|)}\right)=\sum_{i} H_{u}(i, T) / B^{i}$ for each $T$, where $B$ is a big number. Thus, each column in Figure 4.3 will be encoded into one real number so that upper/lower cells in the column correspond to upper/lower bits of the real number. Thanks to the restriction that $G_{u}$ sees only the upper row, the differential equation $h_{u}^{\prime}(t)=g_{u}\left(t, h_{u}(t)\right)$ only needs to cause a weak feedback on $h_{u}$ where each bit of the value of $h_{u}$ affects only much lower bits of its next value. This keeps $g_{u}$ Lipschitz continuous. Now we fill in the details. 
Proof of Lemma 4.1. Let $P, Q,\left(G_{u}\right)_{u},\left(H_{u}\right)_{u}$ be as in Lemma 4.7. By "dividing each unit time into $P(|u|)$ steps," we may assume that for each $T$, there is at most one $i$ such that $G_{u}(i, T, Y) \neq 0$ for some $Y$. Write $j_{u}(T)$ for this unique $i$ (define $j_{u}(T)$ arbitrarily if there is no such $i$ ). We may further assume that

$$
H_{u}\left(i, 2^{Q(|u|)}\right)= \begin{cases}L(u) & \text { if } i=P(|u|) \\ 0 & \text { if } i<P(|u|)\end{cases}
$$

Thus, not only does the bottom right corner of Figure 4.3 equal $L(u)$, as stated already in Lemma 4.7, but we also claim that the other cells in the rightmost column are all 0 . This can be achieved by doubling the time frame and extending $G$ symmetrically so that in the second half it cancels out what it has done. Precisely, we extend $G_{u}$ by

$$
G_{u}\left(i, 2 \cdot 2^{Q(|u|)}-1-T, Y\right)= \begin{cases}0 & \text { if } i=P(|u|)-1 \\ -G_{u}(i, T, Y) & \text { if } i<P(|u|)-1\end{cases}
$$

for $(i, T, Y) \in[P(|u|)] \times\left[2^{Q(|u|)}\right] \times[4]$, and $H_{u}$ by

$$
H_{u}\left(i, 2 \cdot 2^{Q(|u|)}-T\right)= \begin{cases}H_{u}\left(P(|u|), 2^{Q(|u|)}\right) & \text { if } i=P(|u|), \\ H_{u}(i, T) & \text { if } i<P(|u|)\end{cases}
$$

for $(i, T) \in[P(|u|)+1] \times\left[2^{Q(|u|)}+1\right]$, and then add 1 to $Q(|u|)$. It is easy to verify that the equations (4.5) and (4.6) are still satisfied.

Now, assuming (4.8), we construct the families $\left(g_{u}\right)_{u}$ and $\left(h_{u}\right)_{u}$ of Lemma 4.1. For each string $u$ and each $(t, y) \in[0,1] \times[-1,1]$, let $T \in \mathbf{N}, \theta \in[0,1], Y \in \mathbf{Z}, \eta \in[-1 / 4,3 / 4]$ be such that $t=(T+\theta) 2^{-Q(|u|)}$ and $y=(Y+\eta) B^{-j_{u}(T)}$, and define

$$
\begin{aligned}
g_{u}(t, y) & = \begin{cases}\frac{2^{Q(|u|)} \pi \sin (\theta \pi)}{2 B^{j_{u}(T)+1}} G_{u}\left(j_{u}(T), T, Y \bmod 4\right) & \text { if } \eta \leq \frac{1}{4}, \\
\frac{3-4 \eta}{2} g_{u}\left(t, \frac{Y}{B^{j_{u}(T)}}\right)+\frac{4 \eta-1}{2} g_{u}\left(t, \frac{Y+1}{B^{j_{u}(T)}}\right) & \text { if } \eta \geq \frac{1}{4},\end{cases} \\
h_{u}(t) & =\frac{1-\cos (\theta \pi)}{2} \cdot \frac{G_{u}\left(j_{u}(T), T, H_{u}\left(j_{u}(T), T\right)\right)}{B^{j_{u}(T)+1}}+\sum_{i=0}^{P(|u|)} \frac{H_{u}(i, T)}{B^{i}},
\end{aligned}
$$

where $B=2^{\lambda(|u|)+Q(|u|)+5}$. Note that the second branch of (4.11) says that when $\eta \in$ $[1 / 4,3 / 4]$, we define $g_{u}(t, y)$ by interpolating between the nearest two $y$ at which $g_{u}$ is already defined by the first branch. Equation (4.12) says that, when $\theta=0$ (i.e., $t$ is a multiple of $\left.2^{-Q(|u|)}\right)$, the value $h_{u}(t)$ is the real number that encodes the $T$ th column of Figure 4.3 ; as $\theta$ goes from 0 to 1 , it moves to the next value along a cosine curve, whose slope, as we will see below, matches the sine function in the first branch of (4.11). It is easy to verify that the definition is consistent; in particular, we use (4.6) to show that (4.12) stays the same for the two choices of $(T, \theta)$ when $t$ is a multiple of $2^{-Q(|u|)}$.

Conditions (i) and (ii) of Lemma 4.1 are easy to verify. We have (v) with $\rho(k)=$ $P(k)(\lambda(k)+Q(k)+5)$, since $h_{u}(1)=H_{u}\left(P(|u|), 2^{Q(|u|)}\right) / B^{P(|u|)}=L(u) / B^{P(|u|)}=L(u) / 2^{\rho(|u|)}$ by (4.12) and (4.8). Checking the polynomial-time computability of $\left(g_{u}\right)_{u}$ is also routine, using Lemma 2.2 . 
To see (iii), observe that in the right-hand side of (4.12),

o the absolute value of the first term is bounded by $B^{-j_{u}(T)-1} \leq B^{-j_{u}(T)} / 32$,

○ the summands corresponding to $i \leq j_{u}(T)$ are multiples of $B^{-j_{u}(T)}$, and

- the summands corresponding to $i>j_{u}(T)$ are nonnegative numbers, each bounded by $3 / B^{i}=3 B^{-j_{u}(T)} / B^{i-j_{u}(T)} \leq 3 B^{-j_{u}(T)} / 32^{i-j_{u}(T)}$, and thus altogether by $3 B^{-j_{u}(T)} / 31$.

Hence, we can write $h_{u}(t)=(Y+\eta) B^{-j_{u}(T)}$ for some $\eta \in[-1 / 4,1 / 4]$, where

$$
Y=\sum_{i=0}^{j_{u}(T)} H_{u}(i, T) \cdot B^{j_{u}(T)-i} .
$$

Since $B$ is a multiple of 4 , we have $Y \bmod 4=H_{u}\left(j_{u}(T), T\right)$. Substituting these $Y$ and $\eta$ into (the first branch of) (4.11), we get

$$
g_{u}\left(t, h_{u}(t)\right)=\frac{2^{Q(|u|)} \pi \sin (\theta \pi)}{2 B^{j_{u}(T)+1}} G_{u}\left(j_{u}(T), T, H_{u}\left(j_{u}(T), T\right)\right) .
$$

This equals $h_{u}^{\prime}(t)$ calculated from (4.12).

For the Lipschitz condition (iv), note that since the value of $G_{u}$ in the first branch of (4.11) is in $\{-1,0,1\}$, the difference between the two values of $g_{u}$ in the second branch is bounded by $2 \times 2^{Q(|u|)} \pi \sin (\theta \pi) /\left(2 B^{j_{u}(T)+1}\right)<2^{Q(|u|)+2} / B^{j_{u}(T)+1}$. Thus, the slope of $g_{u}$ along the second argument is at most

$$
2 B^{j_{u}(T)} \cdot \frac{2^{Q(|u|)+2}}{B^{j_{u}(T)+1}}=\frac{2^{Q(|u|)+3}}{B} \leq 2^{-\lambda(|u|)}
$$

by our choice of $B$.

\subsection{The discrete initial value problem is hard}

It remains to prove Lemma 4.7. At first sight, our system (4.3)-(4.6) (Figure 4.3) looks too weak to simulate a polynomial-space computation: although we have polynomial amount of memory (rows) and exponential amount of time (columns), the "chains of dependence" of values must run from top to bottom and thus are polynomially bounded in length.

Thus, we give up embedding a general PSPACE computation into Figure 4.3. Instead, we embed another PSPACE-complete problem, QBF (quantified boolean formulas, see Papadimitriou (1994) where it is called QSAT), which asks for the truth value of the given formula $u$ of form

$$
Q_{n} x_{n} \ldots Q_{1} x_{1} \cdot \psi\left(x_{1}, \ldots, x_{n}\right),
$$

where $\psi$ is a boolean formula and $Q_{i} \in\{\forall, \exists\}$ for each $i=1, \ldots, n$.

The truth value of (4.16) is obtained by evaluating a binary tree of depth $n$ whose $2^{n}$ leaves each correspond to an assignment to $\left(x_{1}, \ldots, x_{n}\right)$ and whose internal nodes at level $i$ are labeled $Q_{i}$. This is roughly why it can be simulated by the tableau in Figure 4.3 despite the restriction that the dependence of values must run from top to bottom. We give a formal proof and then an example (Figure 4.4). 
Proof of Lemma 4.7. We may assume that $L=$ QBF. We will construct the polynomials $P, Q$ and families $\left(G_{u}\right)_{u}$ and $\left(H_{u}\right)_{u}$ in Lemma 4.7. Let $u$ be of form (4.16). For each $i=0, \ldots, n$ and $n-i$ bits $b_{i+1}, \ldots, b_{n} \in\{0,1\}$, we write $\psi_{i}\left(b_{i+1}, \ldots, b_{n}\right) \in\{0,1\}$ for the truth value ( 1 for true) of the subformula $Q_{i} x_{i} \ldots Q_{1} x_{1} . \psi\left(x_{1}, \ldots, x_{i}, b_{i+1}, \ldots, b_{n}\right)$, so that $\psi_{0}=\psi$ and $\psi_{n}()=\operatorname{QBF}(u)$. We regard quantifiers as functions from $\{0,1,2,3\}$ to $\{0,1\}$ :

$$
\forall(2)=\exists(2)=\exists(1)=1, \quad \exists(0)=\forall(0)=\forall(1)=0
$$

(the values $\forall(3)$ and $\exists(3)$ do not matter). These correspond to the meaning of the quantifiers: $\forall x$ (resp. $\exists x$ ) means that the subsequent formula is satisfied by 2 (resp. 1 or 2) of the two possible assignments to $x$. Thus,

$$
Q_{i+1}\left(\psi_{i}\left(0, b_{i+2}, \ldots, b_{n}\right)+\psi_{i}\left(1, b_{i+2}, \ldots, b_{n}\right)\right)=\psi_{i+1}\left(b_{i+2}, \ldots, b_{n}\right)
$$

for each $i=0, \ldots, n-1$. For $2 n+1$ bits $b_{0}, \ldots, b_{2 n} \in\{0,1\}$, we write $\overline{b_{2 n} \ldots b_{0}}$ for the number $b_{0}+2 b_{1}+2^{2} b_{2}+\cdots+2^{2 n} b_{2 n}$.

To define $G_{u}$, let

$$
\begin{aligned}
G_{u}\left(i, \overline{T_{2 n} T_{2 n-1} \ldots T_{2 i+2} T_{2 i+1} 100 \ldots 0}, Y\right) \\
=(-1)^{T_{2 i+2}} \times \begin{cases}\psi_{0}\left(T_{1} \oplus T_{2}, T_{3} \oplus T_{4}, \ldots, T_{2 n-1} \oplus T_{2 n}\right) & \text { if } i=0, \\
Q_{i}(Y) & \text { otherwise, }\end{cases}
\end{aligned}
$$

where $\oplus$ denotes the exclusive or; let $G_{u}(i, T, Y)=0$ for other $T$ (that is, when $T$ is not an odd multiple of $2^{2 i}$ ). Define $H_{u}$ from $G_{u}$ by (4.5) and (4.6).

We prove by induction on $i=0, \ldots, n$ that $H_{u}(i, T) \in\{0,1,2\}$ for all $T$, as we mentioned earlier, and that

$$
G_{u}\left(i, S, H_{u}(i, S)\right)=(-1)^{S_{2 i+2}} \psi_{i}\left(S_{2 i+1} \oplus S_{2 i+2}, \ldots, S_{2 n-1} \oplus S_{2 n}\right)
$$

for all $S$ of form $\overline{S_{2 n} S_{2 n-1} \ldots S_{2 i+1} 100 \ldots 0}$ (it is immediate from the definition of $G_{u}$ that $G_{u}\left(i, S, H_{u}(i, S)\right)=0$ for other $\left.S\right)$. Once we have proved this, the case $i=n$ yields $G_{u}\left(n, 2^{2 n}, H_{u}\left(n, 2^{2 n}\right)\right)=\psi_{n}()=\operatorname{QBF}(u)$, and hence $H_{u}\left(n+1,2^{2 n}+1\right)=\operatorname{QBF}(u)$. Since $n<|u|$, we can add dummy rows and columns so that $H_{u}\left(P(|u|), 2^{Q(|u|)}\right)=\mathrm{QBF}(u)$ for some polynomials $P$ and $Q$, as required.

The claims for $i=0$ follow immediately from (4.5) and (4.19). Now suppose (4.20) as the induction hypothesis and fix $T=\overline{T_{2 n} T_{2 n-1} \ldots T_{0}}$. Let $Y=H_{u}(i+1, T)$. By (4.5) and (4.6), we have

$$
Y=\sum_{S=0}^{T-1} G_{u}\left(i, S, H_{u}(i, S)\right) .
$$

Since the assumption (4.20) implies that flipping the two bits $S_{2 i+2}$ and $S_{2 i+1}$ of any $S=\overline{S_{2 n} S_{2 n-1} \ldots S_{0}}$ reverses the sign of $G_{u}\left(i, S, H_{u}(i, S)\right)$, most of the nonzero summands in (4.21) cancel out. The only terms that can survive are those that correspond to $S=\overline{T_{2 n} T_{2 n-1} \ldots T_{2 i+3} 00100 \ldots 0}$ and $S=\overline{T_{2 n} T_{2 n-1} \ldots T_{2 i+3} 01100 \ldots 0}$. This proves $Y \in\{0,1,2\}$. 


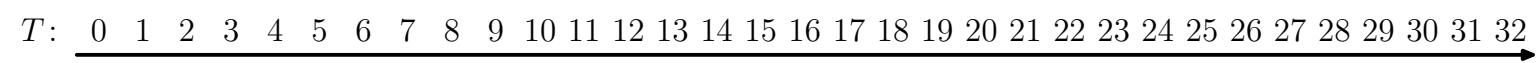

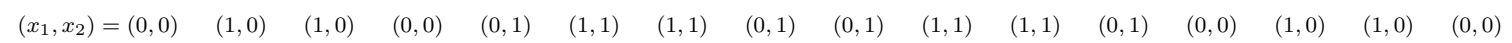

\begin{tabular}{|c|c|c|c|c|c|c|c|c|c|c|c|c|c|c|c|c|c|c|c|c|c|c|c|c|c|c|c|c|c|c|c|c|c|}
\hline$G_{u}(0, T, 0)$ & & +1 & & - & & & & - & & & & & & & & & & + & & + & & -1 & & - & & +0 & & -1 & & -1 & & ( & \\
\hline$H_{u}(1, T)$ & 0 & 0 & 0 & 0 & 1 & 1 & 0 & 0 & 0 & 0 & 1 & 1 & 2 & 2 & 1 & 1 & 0 & 0 & 1 & 1 & 2 & 2 & 1 & 1 & 0 & 0 & 0 & 0 & 1 & 1 & 0 & 0 & 0 \\
\hline$G_{u}(1, T, H$ & $u(1$, & $T)$ & & & + & & & & & & & & & & & & & & & & -1 & & & & & & & & -0 & & & & \\
\hline$H_{u}(2, T)$ & 0 & 0 & 0 & 0 & 0 & 0 & 0 & 0 & 0 & 0 & 0 & 0 & 0 & 1 & 1 & 1 & 1 & 1 & 1 & 1 & 1 & 0 & 0 & 0 & 0 & 0 & 0 & 0 & 0 & 0 & 0 & 0 & 0 \\
\hline$G_{u}(2, T, H$ & $u(2$, & I) & & & & & & & & & & & & & & & & & & & & & & & & & & & & & & & \\
\hline$H_{u}(3, T)$ & 0 & 0 & 0 & 0 & 0 & 0 & 0 & 0 & 0 & 0 & 0 & 0 & 0 & 0 & 0 & 0 & 0 & 1 & 1 & 1 & 1 & 1 & 1 & 1 & 1 & 1 & 1 & 1 & 1 & 1 & 1 & 1 & 1 \\
\hline
\end{tabular}

Figure 4.4: The discrete initial value problem (4.3)-(4.6) corresponding to the formula $u=\exists x_{2} . \forall x_{1} .\left(x_{1} \vee x_{2}\right)$. We follow the convention in Figure 4.3 (but omit the top cells $H_{u}(0, T)$ which are always 0$)$ : the cells contain $H_{u}(i, T)$, and the signed number above the cell $H_{u}(i+1, T)$ indicates the increment $G_{u}\left(i, T, H_{u}(i, T)\right)$ (which is 0 when omitted). The increments $G_{u}(0, T, 0)$ for the top row are determined by the truth values of $x_{1} \vee x_{2}$ for various assignments to $\left(x_{1}, x_{2}\right)$.

When $T=\overline{T_{2 n} T_{2 n-1} \ldots T_{2 i+3} 100 \ldots 0}$, both of these terms survive, so that

$$
Y=\psi_{i}\left(0, T_{2 i+3} \oplus T_{2 i+4}, \ldots, T_{2 n-1} \oplus T_{2 n}\right)+\psi_{i}\left(1, T_{2 i+3} \oplus T_{2 i+4}, \ldots, T_{2 n-1} \oplus T_{2 n}\right) .
$$

Therefore, $Q_{i+1}(Y)=\psi_{i+1}\left(T_{2 i+3} \oplus T_{2 i+4}, \ldots, T_{2 n-1} \oplus T_{2 n}\right)$ by (4.18). Thus,

$$
G_{u}(i+1, T, Y)=(-1)^{T_{2 i+4}} \psi_{i+1}\left(T_{2 i+3} \oplus T_{2 i+4}, \ldots, T_{2 n-1} \oplus T_{2 n}\right)
$$

by (4.19), completing the induction step.

Figure 4.4 shows the table when $u$ be the formula $\exists x_{2} \cdot \forall x_{1} \cdot\left(x_{1} \vee x_{2}\right)$. The values $G_{u}(0, T, 0)$ encode (redundantly) the truth table of the matrix $x_{1} \vee x_{2}$ (first branch of (4.19)). For example, $G_{u}(0, T, 0)= \pm 1$ (resp. 0) for $T=3,5,27,29$ (resp. 1, 7, 25,31) because $\left(x_{1}, x_{2}\right)=(1,0)$ (resp. $\left.(0,0)\right)$ makes $x_{1} \vee x_{2}$ true (resp. false). Also observe that $H_{u}(1, T)$ returns to 0 every eight cells. As a result, the cell $H_{u}(1,4)=1\left(\right.$ resp. $\left.H_{u}(1,12)=2\right)$ represents the fact that when $x_{2}$ is false (resp. true), $x_{1} \vee x_{2}$ is satisfied by one (resp. two) of the assignments to $x_{1}$. Now look at the next row. The second branch of (4.19) says that for odd multiples $T$ of 4 , the values $G_{u}\left(1, T, H_{u}(1, T)\right)$ are \pm 1 or 0 according to whether the upper left cell has a 2 or not. Thus, they encode the smaller truth table for the subformula $\forall x_{1}$. $\left(x_{1} \vee x_{2}\right)$ under each assignment to $x_{2}$. As a result, the cell $H_{u}(2,16)=1$ indicates that this subformula is satisfied by one of the assignments to $x_{2}$, which causes the last row to get incremented at $T=17$. Observe that the final cell $H_{u}(3,32)$ has a 1 , exactly because $u$ is true.

\section{Other versions of the problem}

\subsection{Complexity of the final value}

Ko (1983) discusses another version of the question which relates the complexity of the value $h(1)$, rather than the function $h$, to that of tally languages (subsets of $\{0\}^{*}$ ). 
Definition 2.5 extends straightforwardly to machines taking $d$ oracles. In particular, the case $d=0$ means that a language $L$ is said to reduce to a real number $t$ if there are polynomial-time computable functions $R$ and $S$ such that $L(u)=R(A(S(u)))$ for any string $u$ and any name $A$ of $t$. Now we can state:

Theorem 5.1. For any tally language $L \in$ PSPACE, there are functions $g$ and $h$ satisfying $(*)$ such that $L$ reduces to $h(1)$.

This can be proved by arranging the building blocks $g_{u}$ from Lemma 4.1, as we did for Theorem 3.2, but in a different way. See Section 6.2 for details.

As a corollary to Theorem 5.1, all tally languages of PSPACE are in $\mathbf{P}$ if $(*)$ implies that $h(1)$ is polynomial-time computable. This improves on Theorem 4 of Ko (1983), which showed the same thing with the Lipschitz condition replaced by a weaker condition.

\section{$5.2 \quad$ Volterra integral equations}

Ko (1992) later studied the complexity of Volterra integral equations of the second kind

$$
h(t)=f(t)+\int_{0}^{t} g(t, \tau, h(\tau)) \mathrm{d} \tau, \quad t \in[0,1]
$$

where function $h$ is to be solved from given $f$ and $g$. As before, we suppose that $f$ and $g$ are polynomial-time computable and ask how complex $h$ can be.

If $g$ is Lipschitz continuous (in its last argument), $h$ is polynomial-space computable by Picard's iteration method (Section 3 of Ko (1992)). On the other hand, the best lower bound (in the sense analogous to the "if" direction of Corollary 3.3) has been $\mathbf{P} \# \mathbf{P}$. One of the two open problems in Ko (1992) was to close this gap. Our Theorem 3.2 has solved it, because the initial value problem (1.1) is the special case of (5.2) where $f$ is constantly zero and $g$ ignores its first argument:

Corollary 5.3. There are functions $f:[0,1] \rightarrow \mathbf{R}$ and $g:[0,1] \times[0,1] \times \mathbf{R} \rightarrow \mathbf{R}$ such that $g$ is Lipschitz continuous (in its last argument), $f$ and $g$ are both polynomial-time computable, and the (unique) solution of (5.2) is polynomial-space complete.

The other problem was about the following weak version of the Lipschitz condition (1.2):

$$
\left|g\left(t, y_{0}\right)-g\left(t, y_{1}\right)\right| \leq 2^{r(n)}\left|y_{0}-y_{1}\right|, \quad n \in \mathbf{N}, t \in\left[0,1-2^{-n}\right], y_{0}, y_{1} \in \mathbf{R},
$$

where $r$ is a polynomial. Assuming $(*)$ with (1.2) replaced by (5.4), how complex can $h$ be, provided it has a polynomial modulus of continuity? Ko (1992) asked this question for the Volterra equation (5.2) (in which case the $g$ in (5.4) takes one more argument), and showed that $h$ is exponential-space computable and can be EXPTIME-hard. His second open problem was to close this gap.

The motivation for this problem comes from Volterra integral equations of the first kind, a class of equations that are considered harder to solve than (5.2). A common approach to solve them is to convert the equation into the form (5.2). This conversion does not 
preserve Lipschitz continuity (1.2); the new equation merely satisfies (5.4). See Ko (1992) for details.

The following theorem settles this problem (see Section 6.3 for a proof, again using Lemma 4.1). In fact, we have EXPSPACE-completeness even for the simple differential equation (1.1):

THEOREM 5.5. There are functions $g$ and $h$ satisfying $(*)$ with (1.2) replaced with (5.4) such that $h$ has a polynomial modulus of continuity and yet is EXPSPACE-hard.

Note, however, that this theorem says nothing directly about the complexity of Volterra equations of the first kind. It merely addresses the complexity of a class of equations that may arise in a particular approach to solving them.

\section{Putting the building blocks together}

We give the details of the proofs of Theorems 3.2, 5.1 and 5.5 from Lemma 4.1.

\subsection{Proof of Theorem 3.2}

The rough ideas are explained in Section 4.1. Let $L$ be a PSPACE-complete language. Use Lemma 4.1 for $\lambda(k)=2 k+2$ to obtain polynomial $\rho$ and families $\left(g_{u}\right)_{u},\left(h_{u}\right)_{u}$. Since $\left(g_{u}\right)_{u}$ is polynomial-time computable, there is a polynomial $\gamma$ satisfying $\left|g_{u}(t, y)\right| \leq 2^{\gamma(|u|)-|u|}$. For each binary string $u$, let $\Lambda_{u}=2^{\lambda(|u|)}, \Gamma_{u}=2^{\gamma(|u|)}$ and

$$
c_{u}=1-\frac{1}{2^{|u|}}+\frac{2 \bar{u}+1}{\Lambda_{u}}, \quad \quad l_{u}^{\mp}=c_{u} \mp \frac{1}{\Lambda_{u}},
$$

where $\bar{u} \in\left\{0, \ldots, 2^{|u|}-1\right\}$ is $u$ read as an integer in binary notation. This divides $[0,1)$ into intervals $\left[l_{u}^{-}, l_{u}^{+}\right]$indexed by $u \in\{0,1\}^{*}$. Define

$$
\begin{aligned}
g\left(l_{u}^{\mp} \pm \frac{t}{\Lambda_{u}}, \frac{y}{\Lambda_{u} \Gamma_{u}}\right) & = \pm \frac{g_{u}(t, \hat{y})}{\Gamma_{u}}, \\
h\left(l_{u}^{\mp} \pm \frac{t}{\Lambda_{u}}\right) & =\frac{h_{u}(t)}{\Lambda_{u} \Gamma_{u}}
\end{aligned}
$$

for each $t \in[0,1]$ and $y \in \mathbf{R}$, where $\hat{y}=\max \{-1, \min \{1, y\}\}$. Let $g(1, y)=h(1)=0$ for each $y \in \mathbf{R}$. These define $g$ and $h$ "seamlessly" by Lemma 4.1(ii).

We show that $g$ and $h$ satisfy $(*)$. We begin with equation (1.1). It is easy to see that $h(0)=0$ and $h^{\prime}(1)=0=g(1, h(1))$. Since any number in $[0,1)$ can be written in the form $l_{u}^{\mp} \pm t / \Lambda_{u}$ for some $u$ and $t \in[0,1]$, we have (1.1) by

$$
\begin{aligned}
h^{\prime}\left(l_{u}^{\mp} \pm \frac{t}{\Lambda_{u}}\right) & = \pm \frac{h_{u}^{\prime}(t)}{\Gamma_{u}}= \pm \frac{g_{u}\left(t, h_{u}(t)\right)}{\Gamma_{u}} \\
& =g\left(l_{u}^{\mp} \pm \frac{t}{\Lambda_{u}}, \frac{h_{u}(t)}{\Lambda_{u} \Gamma_{u}}\right)=g\left(l_{u}^{\mp} \pm \frac{t}{\Lambda_{u}}, h\left(l_{u}^{\mp} \pm \frac{t}{\Lambda_{u}}\right)\right),
\end{aligned}
$$


where equalities are by (6.3), (iii), (6.2), (6.3), respectively.

The Lipschitz condition (1.2) is satisfied with $Z=1$ by (iv) and our choice of $\lambda$. To see that $g$ is polynomial-time computable, we use (the obvious two-dimensional version of) Lemma 2.2. When asked for a $2^{-m}$-approximation of $g(T, Y)$ for rational numbers $T$ and $Y$, the machine can find $u, \pm, t, y$ with $(T, Y)=\left(l_{u}^{\mp} \pm t / \Lambda_{u}, y / \Lambda_{u} \Gamma_{u}\right)$ in polynomial time. Since (6.2) lies in $\left[-2^{-|u|}, 2^{-|u|}\right]$, the machine can safely answer 0 if $m<|u|$. Otherwise it can answer by computing $g_{u}(t, \hat{y})$ with precision $2^{-m}$, which can be done in time polynomial in $m+|u| \leq 2 m$ by the polynomial-time computability of $\left(g_{u}\right)_{u}$.

We have thus proved $(*)$. Since

$$
h\left(c_{u}\right)=\frac{h_{u}(1)}{\Lambda_{u} \Gamma_{u}}=\frac{L(u)}{2^{\lambda(|u|)+\gamma(|u|)+\rho(|u|)}}
$$

by (6.3) and (v), the problem $L$ reduces to $h$. More precisely, the functions $R, S, T$ in Definition 2.5 can be given by

$$
\begin{aligned}
R(u, v) & = \begin{cases}0 & \text { if } v \text { denotes } 0 \\
1 & \text { if } v \text { denotes } 1,\end{cases} \\
S\left(u, 0^{n}\right) & =\text { a string denoting }\left\lfloor 2^{n} c_{u}\right\rfloor, \\
T(u) & =0^{\lambda(|u|)+\gamma(|u|)+\rho(|u|)} .
\end{aligned}
$$

Since $L$ is PSPACE-complete, so is $h$.

\subsection{Proof of Theorem 5.1}

We will construct $g$ and $h$ satisfying $(*)$ such that the tally language $L \in$ PSPACE reduces to $h(1)$. Apply Lemma 4.1 to $\lambda(k)=k+1$ to obtain the polynomial $\rho$ and the families $\left(g_{u}\right)_{u},\left(h_{u}\right)_{u}$. As we did for Theorem 3.2, we are going to divide [0,1) into countably many intervals $\left[l_{k}, l_{k+1}\right]$ and put there some copies of the block $g_{0^{k}}$ of Lemma 4.1 ; but this time, we do not put the mirror reflection to bring $h$ back to 0 (Figure 6.1). The values $h_{u}(1)$ pile up, so that we can recover any of them by looking at $h(1)$.

Since $\left(g_{u}\right)_{u}$ is polynomial-time computable, there is a monotone polynomial $\gamma: \mathbf{N} \rightarrow \mathbf{N}$ satisfying $\left|g_{0^{k}}(t, y)\right| \leq 2^{\gamma(k)}$ for each $k$. Let $l_{k}=1-2^{-k}$ and define

$$
\begin{gathered}
g\left(l_{k}+\frac{t}{2^{k+1}}, \frac{2 j+(-1)^{j} y}{2^{2 k+\gamma(k)+\bar{\rho}(k)}}\right)=\frac{g_{0^{k}}(t, y)}{2^{k-1+\gamma(k)+\bar{\rho}(k)}}, \\
h\left(l_{k}+\frac{t}{2^{k+1}}\right)=\frac{h_{0^{k}}(t)}{2^{2 k+\gamma(k)+\bar{\rho}(k)}}+\sum_{\kappa=0}^{k-1} \frac{L\left(0^{\kappa}\right)}{2^{2 \kappa+\gamma(\kappa)+\bar{\rho}(\kappa)+\rho(\kappa)}}
\end{gathered}
$$

for each $k \in \mathbf{N}, t \in[0,1], y \in[-1,1]$ and $j \in \mathbf{Z}$, where $\bar{\rho}(k)=\rho(0)+\cdots+\rho(k-1)$. Complete the definition by $g(1, y)=0$ and

$$
h(1)=\sum_{k=0}^{\infty} \frac{L\left(0^{k}\right)}{2^{2 k+\gamma(k)+\bar{\rho}(k)+\rho(k)}} .
$$




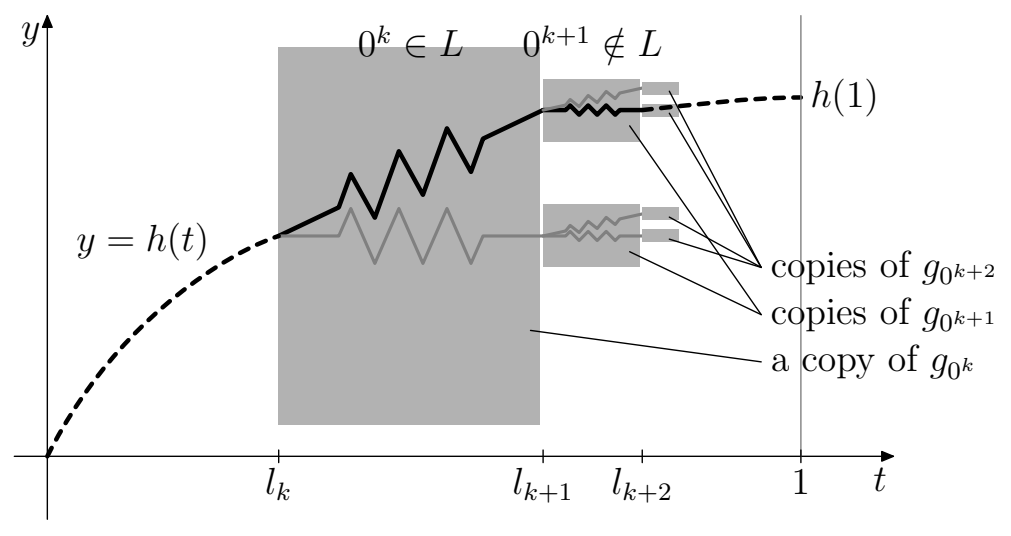

Figure 6.1: Compare with Figure 4.1. The blocks $g_{0^{k}}$ are now stacked vertically so that the tally language $L$ can be recovered from $h(1)$.

By (6.11), the language $L$ reduces to $h(1)$.

We show that these $g$ and $h$ satisfy $(*)$. Well-definedness and Lipschitz continuity of $g$ follow from (ii) and (iv) of Lemma 4.1, similarly to the proof of Theorem 3.2. Polynomialtime computability also follows from that of $\left(g_{u}\right)_{u}$ again. Since all terms under the summation symbol in (6.10) are divisible by $4 / 2^{2 k+\gamma(k)+\bar{\rho}(k)}$, substituting (6.10) into the second argument of (6.9) yields

$$
g\left(l_{k}+\frac{t}{2^{k+1}}, h\left(l_{k}+\frac{t}{2^{k+1}}\right)\right)=\frac{g_{0^{k}}\left(t, h_{0^{k}}(t)\right)}{2^{k-1+\gamma(k)+\bar{\rho}(k)}}=\frac{h_{0^{k}}^{\prime}(t)}{2^{k-1+\gamma(k)+\bar{\rho}(k)}}=h^{\prime}\left(l_{k}+\frac{t}{2^{k+1}}\right)
$$

where the second and third equalities are by (iii) and (6.10), respectively. This and $h^{\prime}(1)=$ $0=g(h(1))$ yield (1.1). We have proved $(*)$.

\subsection{Proof of Theorem 5.5}

Let $L \in$ PSPACE be the set of triples $\left(M, x, 0^{s}\right)$ such that $M$ encodes a Turing machine that, on string input $x$, uses at most $s$ tape cells and accepts. For each triple $w=(M, x, s)$, let $u_{w}=\left(M, x, 0^{s}\right)$. With a suitable encoding, we have $\left|u_{w}\right| \leq 2^{|w|}$. It is easy to see that $L^{\prime}=\left\{w: u_{w} \in L\right\}$ is EXPSPACE-complete.

The desired $g$ and $h$ such that $L^{\prime}$ reduces to $h$ will be constructed as follows. As we did for Theorem 3.2, we divide $[0,1)$ into infinitely many intervals $\left[l_{w}^{-}, l_{w}^{+}\right]$with midpoints $c_{w}$, and put there the functions $g_{u_{w}}$ of Lemma 4.1 to compute whether $u_{w} \in L$, which is equivalent to $w \in L^{\prime}$. But this time, the outcome $h_{u_{w}}(1)$ is exponentially small in $|w|$ (even when it is positive), so we need "amplifiers" to make the value $h\left(c_{w}\right)$ visibly large (Figure 6.2). Because we use stronger and stronger amplifiers as $|w| \rightarrow \infty$, the function $g$ will not satisfy the full Lipschitz condition (1.2), but it still satisfies the weaker condition (5.4).

Now we fill in the details. Apply Lemma 4.1 to $\lambda(k)=0$ (and the above $L$ ) to obtain the polynomial $\rho$ and families $\left(g_{u}\right)_{u},\left(h_{u}\right)_{u}$. Since $\left(g_{u}\right)_{u}$ is polynomial-time computable, there is a polynomial $\gamma$ satisfying $\left|g_{u}(t, y)\right| \leq 2^{\gamma(|u|)-|u|}$. We may assume that $\gamma(k)-k$ is strictly increasing in $k$ and that $(1.5 \ln 2) \gamma(k) \leq 2^{\gamma(k)-k}$ for all $k$. For each binary string $w$, 


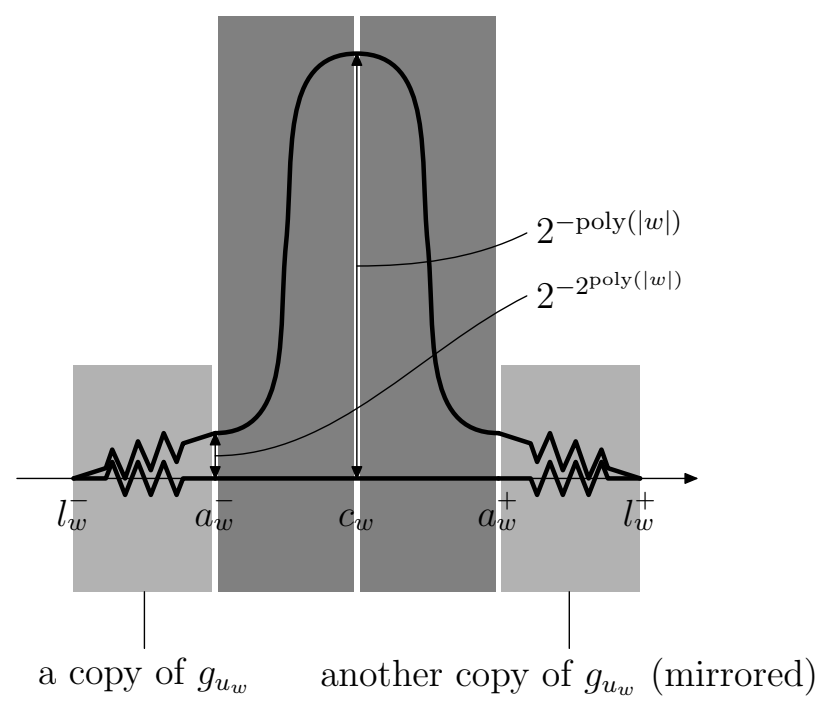

Figure 6.2: The construction for Theorem 5.5 is similar to Figure 4.1, but this time we put an "amplifier" in the middle. Then $w \in L$ if and only if $h\left(c_{w}\right)$ is positive, and in this case it is only polynomially small in $|w|$.

let $\Lambda_{w}=2^{2|w|+3}, \Gamma_{w}=2^{\gamma\left(2^{|w|}\right)}$ and

$$
c_{w}=1-\frac{1}{2^{|w|}}+\frac{4 \bar{w}+2}{\Lambda_{w}}, \quad a_{w}^{\mp}=c_{w} \mp \frac{1}{\Lambda_{w}}, \quad l_{w}^{\mp}=c_{w} \mp \frac{2}{\Lambda_{w}},
$$

where $\bar{w} \in\left\{0, \ldots, 2^{|w|}-1\right\}$ is $w$ read as an integer in binary notation. This divides $[0,1)$ into intervals $\left[l_{w}^{-}, l_{w}^{+}\right]$of length $4 / \Lambda_{w}$ indexed by $w \in\{0,1\}^{*}$. Define

$$
\begin{aligned}
g\left(l_{w}^{\mp} \pm \frac{t}{\Lambda_{w}}, \frac{y}{\Lambda_{w} \Gamma_{w}}\right) & = \pm \frac{g_{u_{w}}(t, \hat{y})}{\Gamma_{w}} \\
g\left(a_{w}^{\mp} \pm \frac{t}{\Lambda_{w}}, Y\right) & = \pm 6 t(1-t) Y \Lambda_{w} \ln \Gamma_{w} \\
h\left(l_{w}^{\mp} \pm \frac{t}{\Lambda_{w}}\right) & =\frac{h_{u_{w}}(t)}{\Lambda_{w} \Gamma_{w}} \\
h\left(a_{w}^{\mp} \pm \frac{t}{\Lambda_{w}}\right) & =\frac{\Gamma_{w}^{t^{2}(3-2 t)} h_{u_{w}}(1)}{\Lambda_{w} \Gamma_{w}}
\end{aligned}
$$

for each $t \in[0,1]$ and $y, Y \in \mathbf{R}$, where $\hat{y}=\max \{-1, \min \{1, y\}\}$. Let $g(1, Y)=h(1)=0$ for each $Y \in \mathbf{R}$. This defines $g$ and $h$ seamlessly by Lemma 4.1(ii). Recall the idea explained in Figure 6.2: the equations (6.14) and (6.16) are analogous to (6.2) and (6.3) in the proof of the main theorem, and (6.15) and (6.17) stand for the magnifier.

We show that $g$ and $h$ satisfy $(*)$ with $(1.2)$ replaced by (5.4).

For the equation (1.1), it is again easy to see that $h(0)=0$ and $h^{\prime}(1)=0=g(1, h(1))$. Numbers in $[0,1)$ can be written either as $l_{w}^{\mp} \pm t / \Lambda_{w}$ or as $a_{w}^{\mp} \pm t / \Lambda_{w}$, and for them the 
equation follows respectively by

$$
\begin{aligned}
h^{\prime}\left(l_{w}^{\mp} \pm \frac{t}{\Lambda_{w}}\right) & = \pm \frac{h_{u_{w}}^{\prime}(t)}{\Gamma_{w}}= \pm \frac{g_{u_{w}}\left(t, h_{u_{w}}(t)\right)}{\Gamma_{w}} \\
= & g\left(l_{w}^{\mp} \pm \frac{t}{\Lambda_{w}}, \frac{h_{u_{w}}(t)}{\Lambda_{w} \Gamma_{w}}\right)=g\left(l_{w}^{\mp} \pm \frac{t}{\Lambda_{w}}, h\left(l_{w}^{\mp} \pm \frac{t}{\Lambda_{w}}\right)\right), \\
h^{\prime}\left(a_{w}^{\mp} \pm \frac{t}{\Lambda_{w}}\right) & = \pm \frac{6 t(1-t) \ln \Gamma_{w}}{\Gamma_{w}} \Gamma_{w}^{t^{2}(3-2 t)} h_{u_{w}}(1) \\
= & g\left(a_{w}^{\mp} \pm \frac{t}{\Lambda_{w}}, \frac{\Gamma_{w}^{t^{2}(3-2 t)} h_{u_{w}}(1)}{\Lambda_{w} \Gamma_{w}}\right)=g\left(a_{w}^{\mp} \pm \frac{t}{\Lambda_{w}}, h\left(a_{w}^{\mp} \pm \frac{t}{\Lambda_{w}}\right)\right),
\end{aligned}
$$

where we used (6.16), (iii), (6.14), (6.16), (6.17), (6.15), (6.17) for each equality.

The condition (5.4) is satisfied with $r(k)=2 k+3+s(k)$, where $s$ is any polynomial such that $2^{s(k)} \geq(1.5 \ln 2) \gamma\left(2^{k}\right)$. For if $T \in\left[l_{w}^{-}, a_{w}^{-}\right]$or $T \in\left[a_{w}^{+}, l_{w}^{+}\right]$for some $w$, then by (iv) and (6.14), we have

$$
\frac{\left|g\left(T, Y_{0}\right)-g\left(T, Y_{1}\right)\right|}{\left|Y_{0}-Y_{1}\right|} \leq \frac{2^{-\lambda\left(\left|u_{w}\right|\right)}}{\Gamma_{w}} \Lambda_{w} \Gamma_{w} \leq \Lambda_{w}=2^{2|w|+3} \leq 2^{r(|w|)} .
$$

If $T \in\left[a_{w}^{-}, a_{w}^{+}\right]$for some $w$, then by $(6.15)$ we have

$$
\begin{aligned}
\frac{\left|g\left(T, Y_{0}\right)-g\left(T, Y_{1}\right)\right|}{\left|Y_{0}-Y_{1}\right|} \leq 2^{2|w|+3} & \cdot 1.5 \ln \Gamma_{w} \\
& =2^{2|w|+3} \cdot 1.5 \gamma\left(2^{|w|}\right) \ln 2 \leq 2^{2|w|+3} \cdot 2^{s(|w|)}=2^{r(|w|)} .
\end{aligned}
$$

To see that $g$ is polynomial-time computable, we use the characterization in (the obvious two-dimensional generalization of) Lemma 2.2. Suppose we are asked for an approximation of $g(T, Y)$ to precision $2^{-m}$ for some $T \in[0,1] \cap \mathbf{Q}$ and $Y \in[-1,1] \cap \mathbf{Q}$. We first find a string $w$ and $t \in[0,1] \cap \mathbf{Q}$ such that $T$ can be written as $l_{w}^{\mp} \pm t / \Lambda_{w}$ or as $a_{w}^{\mp} \pm t / \Lambda_{w}$. In the latter case, it is easy to compute the desired approximation using (6.15). In the former case, we use (6.14) as follows:

○ If $m<2^{|w|}$, we can safely answer 0 , because the value $(6.14)$ is in $\left[-2^{-2^{|w|}}, 2^{-2^{|w|}}\right]$ by $\left|g_{u_{w}}(t, \hat{y})\right| \leq 2^{\gamma\left(\left|u_{w}\right|\right)-\left|u_{w}\right|} \leq 2^{\gamma\left(2^{|w|}\right)-2^{|w|}}$.

○ Otherwise, we compute $\hat{y} \in \mathbf{Q}$, where $y=\Lambda_{w} \Gamma_{w} Y$, and then get the desired approximation of $(6.14)$ by computing $g_{u_{w}}(t, \hat{y})$ to an appropriate precision. This can be done, by the polynomial-time computability of $\left(g_{u}\right)_{u}$, in time polynomial in $m$ and $\left|u_{w}\right|$. But this is in fact polynomial in $m$, since $\left|u_{w}\right| \leq 2^{|w|} \leq m$.

We have thus proved $(*)$ with (1.2) replaced by (5.4). Since (6.17) yields

$$
h\left(c_{w}\right)=\frac{h_{u_{w}}(1)}{\Lambda_{w}}=\frac{L\left(u_{w}\right)}{2^{2|w|+3+\rho(|w|)}},
$$

the language $L^{\prime}$ reduces to $h$. Since $L^{\prime}$ is EXPSPACE-complete, $h$ is EXPSPACE-hard.

Finally, we claim that $h$ has a polynomial modulus of continuity. Precisely, we show that $\left|h\left(T_{0}\right)-h\left(T_{1}\right)\right|<2^{-k}$ whenever $k \in \mathbf{N}$ and $0 \leq T_{1}-T_{0}<2^{-s(k)-k}$. 
○ If $T_{1} \geq 1-2^{-k}$, then $T_{1} \in\left[l_{w}^{-}, l_{w}^{+}\right]$for some $w$ of length $\geq k$, so $\left|h_{1}\left(T_{1}\right)\right| \leq 1 / \Lambda_{w} \leq$ $1 / 2^{2 k+3}$ by (6.16), (6.17) and (i). Applying the same argument to $T_{0}>T_{1}-2^{-s(k)-k} \geq$ $1-2^{-(k-1)}$, we obtain $\left|h_{1}\left(T_{0}\right)\right| \leq 1 / 2^{2(k-1)+3}$. Thus, $\left|h\left(T_{0}\right)-h\left(T_{1}\right)\right| \leq\left|h\left(T_{0}\right)\right|+$ $\left|h\left(T_{1}\right)\right| \leq 1 / 2^{2 k+3}+1 / 2^{2(k-1)+3}<2^{-k}$.

○ If $T_{1} \leq 1-2^{-k}$, then each point $T \in\left[T_{0}, T_{1}\right]$ belongs to $\left[l_{w}^{-}, l_{w}^{+}\right]$for some $w$ of length $<k$. If $T \in\left[l_{w}^{-}, a_{w}^{-}\right] \cup\left[a_{w}^{+}, l_{w}^{+}\right]$, then $\left|h^{\prime}(T)\right| \leq 2^{\gamma\left(\left|u_{w}\right|\right)} / \Gamma_{w} \leq 1$ by the first line of (6.18); otherwise, $\left|h^{\prime}(T)\right| \leq 6(1 / 4)\left(\ln \Gamma_{w}\right) h_{u_{w}}(1)=(1.5 \ln 2) \gamma\left(2^{|w|}\right) h_{u_{w}}(1) \leq 2^{s(|w|)} \leq 2^{s(k)}$ by the first line of (6.19). We thus have $\left|h^{\prime}(T)\right| \leq 2^{s(k)}$, and hence $\left|h\left(T_{0}\right)-h\left(T_{1}\right)\right| \leq$ $2^{s(k)}\left(T_{1}-T_{0}\right)<2^{s(k)} \cdot 2^{-s(k)-k}=2^{-k}$.

\section{Related results and open problems}

\subsection{Other results on differential equations}

Table 7.1 summarizes what is known about the computability and complexity of the initial value problem (1.1) in our sense. Computability of other aspects of the solution is discussed by Cenzer \& Remmel (2004), Graça et al. (2008) and Kawamura (2009a). Edalat \& Pattinson (2004) give a domain-theoretic account for the problem.

The status between the last two rows of Table 7.1 remains open. What happens, for example, if we assume that $g$ is infinitely often differentiable?

Computability (or not) of other classes of differential equations is studied by Pour-El \& Richards (1981), Pour-El \& Zhong (1997), Gay et al. (2001), Weihrauch \& Zhong (2002), Weihrauch \& Zhong (2006) and Zhong (2007). Less is known about their computational complexity.

Table 7.1: Assuming that $g$ is polynomial-time computable, how complex can the solution $h$ of (1.1) be?

\begin{tabular}{|c|c|c|}
\hline Assumptions & Upper bounds & Lower bounds \\
\hline None & - & $\begin{array}{l}\text { can be (non-unique and) all non-computable: } \\
\text { Aberth (1971), Pour-El \& Richards (1979), } \\
\text { Ko (1983) }\end{array}$ \\
\hline $\begin{array}{l}h \text { is the unique } \\
\text { solution }\end{array}$ & $\begin{array}{l}\text { computable: Osgood (1898), } \\
\text { Pour-El \& Richards (1979) }\end{array}$ & $\begin{array}{l}\text { can take arbitrarily long time: Miller (1970), } \\
\text { Ko (1983) }\end{array}$ \\
\hline condition (5.4) & exponential-space: Ko (1992) & can be EXPSPACE-hard (our Theorem 5.5) \\
\hline $\begin{array}{l}\text { the Lipschitz } \\
\text { condition (1.2) }\end{array}$ & polynomial-space: Ko (1983) & can be PSPACE-hard (our Theorem 3.2) \\
\hline$g$ is analytic & $\begin{array}{l}\text { polynomial-time: } \\
\text { Ko \& Friedman (1988), } \\
\text { Kawamura (2010a) }\end{array}$ & - \\
\hline
\end{tabular}




\subsection{Constructive versions}

A reasonable criticism about the results in Table 7.1 is that they deal with the complexity of each single solution $h$, whereas the practical concern for numerical analysts would be the complexity of the operator that "computes $h$ from $g$." For computability, such constructive formulation is possible through a suitable representation of the function space (see Chapter 6 of Weihrauch (2000)). For complexity, formulation of constructive results requires some more ideas, and is undertaken in a recent work of Kawamura \& Cook (2010b).

\section{Acknowledgements}

The author is grateful to Stephen A. Cook, Ker-I Ko and the anonymous referees for comments on the manuscript that helped improve the presentation.

This work was supported in part by the Nakajima Foundation and by the Natural Sciences and Engineering Research Council of Canada.

A preliminary version of this work appeared as Kawamura (2009b).

\section{References}

O. Aberth (1971). The Failure in Computable Analysis of a Classical Existence Theorem for Differential Equations. Proceedings of the American Mathematical Society 30, 151-156.

P. Beame, S. Cook, J. Edmonds, R. Impagliazzo \& T. Pitassi (1998). The Relative Complexity of NP Search Problems. Journal of Computer and System Sciences 57(1), 3-19.

V. Brattka, P. Hertling \& K. Weihrauch (2008). A Tutorial on Computable Analysis. In New Computational Paradigms: Changing Conceptions of What is Computable, S. B. Cooper, B. LÖwE \& A. SorBI, editors, 425-491. Springer.

D. Cenzer \& J. B. Remmel (2004). Index Sets for Computable Differential Equations. Mathematical Logic Quarterly 50(4-5), 329-344.

A. Edalat \& D. Pattinson (2004). A Domain Theoretic Account of Picard's Theorem. In Proceedings of the 31st International Colloquium on Automata, Languages and Programming, volume 3142 of Lecture Notes in Computer Science, 494-505.

H. Friedman (1984). The Computational Complexity of Maximization and Integration. Advances in Mathematics 53, 80-98.

W. Gay, B. Zhang \& N. Zhong (2001). Computability of Solutions of the Korteweg-de Vries Equation. Mathematical Logic Quarterly 47(1), 93-110.

D. S. Graça, J. Buescu \& M. L. Campagnolo (2008). Boundedness of the Domain of Definition is Undecidable for Polynomial ODEs. In Proceedings of the Fourth International Conference on Computability and Complexity in Analysis, volume 202 of Electronic Notes in Theoretical Computer Science, 49-57.

A. Grzegorczyk (1955). Computable Functionals. Fundamenta Mathematicae 42, 168-202. 
H. J. Hoover (1990). Feasible Real Functions and Arithmetic Circuits. SIAM Journal on Computing 19(1), 182-204.

A. Kawamura (2009a). Differential Recursion. ACM Transactions on Computational Logic 10(3), Article 22.

A. Kawamura (2009b). Lipschitz Continuous Ordinary Differential Equations are PolynomialSpace Complete. In Proceedings of the 24th Annual IEEE Conference of Computational Complexity, 149-160.

A. Kawamura (2010a). Complexity of Initial Value Problems. Manuscript.

A. Kawamura \& S. Cook (2010b). Complexity Theory for Operators in Analysis. To appear in Proceedings of the 42nd ACM Symposium on Theory of Computing.

K. Ko (1983). On the Computational Complexity of Ordinary Differential Equations. Information and Control 58, 157-194.

K. Ko (1991). Computational Complexity of Real Functions. Birkhäuser Boston.

K. Ko (1992). On the Computational Complexity of Integral Equations. Annals of Pure and Applied Logic 58(3), 201-228.

K. Ko \& H. Friedman (1982). Computational Complexity of Real Functions. Theoretical Computer Science 20(3), 323-352.

K. Ko \& H. Friedman (1988). Computing Power Series in Polynomial Time. Advances in Applied Mathematics 9, 40-50.

W. Miller (1970). Recursive Function Theory and Numerical Analysis. Journal of Computer and System Sciences 4, 465-472.

W. F. Osgood (1898). Beweis der Existenz einer Lösung der Differentialgleichung $\frac{d y}{d x}=f(x, y)$ ohne Hinzunahme der Cauchy-Lipschitz'schen Bedingung. Monatshefte für Mathematik 9(1), $331-345$.

C. H. Papadimitriou (1994). Computational Complexity. Addison Wesley.

M. B. Pour-El \& I. Richards (1979). A Computable Ordinary Differential Equation Which Possesses No Computable Solution. Annals of Mathematical Logic 17(1-2), 61-90.

M. B. Pour-El \& I. Richards (1981). The Wave Equation with Computable Initial Data such that its Unique Solution is not Computable. Advances in Mathematics 39(3), 215-239.

M. B. Pour-El \& N. Zhong (1997). The Wave Equation with Computable Initial Data Whose Unique Solution is Nowhere Computable. Mathematical Logic Quarterly 43, 499-509.

K. Weinrauch (2000). Computable Analysis: An Introduction. Texts in Theoretical Computer Science. Springer.

K. Weihrauch \& N. Zhong (2002). Is Wave Propagation Computable or Can Wave Computers Beat the Turing Machine? Proceedings of the London Mathematical Society 85(2), 312-332.

K. Weinrauch \& N. Zhong (2006). Computing Schrödinger Propagators on Type-2 Turing Machines. Journal of Complexity 22(6), 918-935.

N. Zhong (2007). Computable Analysis of a Boundary-Value Problem for the Korteweg-de Vries Equation. Theory of Computing Systems 41(1), 155-175. 